\title{
DUALIDAD, LA LOCOMOTORA MINERA Vs. EL PULMÓN NEGRO
}

\author{
Beatriz Pescador Vargas ${ }^{1}$, Laura Alejandra Roa Culma ${ }^{2}$ \\ ${ }^{1}$ Magister en Biología, Docente Asociada Facultad de Medicina - Universidad Militar Nueva Granada \\ ${ }^{2}$ Bióloga y Estudiante Facultad de Medicina
}

Recibido: 3 Junio 2016 Aceptado: 6 Julio 2016

Sombrios días de socavón, noches de tragedia, desesperanza y desilución, se sienten en mi alma. Así mi vida pasando voy,

porque minero soy.... "el minero" Jaime Medinaceli

\section{RESUMEN}

La neumoconiosis es caracterizada por el depósito nodular difuso de polvo en los pulmones como resultado de la exposición prolongada a polvo bituminoso o de antracita en los trabajadores de las minas de carbón. La neumoconiosis de los mineros del carbón también se denomina enfermedad del pulmón negro o antracosis. Un minero del carbón que padece o desarrolla una Antracosis puede presentar numerosos nódulos redondeados pulmonares en poco tiempo. Dichos nódulos aparecen en ocasiones en ausencia de una antracosis simple. A nivel histológico pueden parecerse a los nódulos reumatoides, pero tienen una zona periférica de inflamación aguda. Estos nódulos representan la respuesta inmunológica a la diátesis reumatoide asociada.

En Colombia la minería es un factor estratégico a nivel económico para su desarrollo. Sin embargo, existen factores negativos derivados de ésta que giran en una carrera sin control ni reglas claras; esta actividad hace trámite en el territorio, arrastrando una estela de problemas sobre la sociedad, el ambiente, el bienestar y la salud de las personas.

Palabras clave: Neumoconiosis, Antracosis, Nódulos de carbón, Disnea, Macrófagos Alveolares. 


\title{
DUALITY, MINING LOCOMOTIVE VS. BLACK LUNG
}

\begin{abstract}
Pneumoconiosis is characterized by diffuse nodular dust in the lungs as a result of prolonged exposure of workers in coal mines to bituminous dust or anthracite. Pneumoconiosis presented in coal miners is also called black lung disease lung. A coal miner who develops Anthracosis can present numerous pulmonary rounded nodules in a short time. These nodules appear sometimes in the absence of a simple anthracosis. Histologically they may look like resemble rheumatoid nodules, with the difference; they have a peripheral area of acute inflammation. These nodes represent the immune response associated to rheumatoid diathesis.

In Colombia, mining is a strategy for economical development. However, there are negative factors arising rotating it in a race without control or clear rules; This activity is pending in the territory, dragging a trail of problems on society, the environment, welfare and People's health
\end{abstract}

Keywords: Pneumoconiosis, Black Lung, Coal nodules, Dyspnoea, Alveolar macrophages.

\section{DUALIDADE, LOCOMOTIVA MINERA Vs. PULMÃO PRETO}

\section{RESUMO}

A pneumoconiose é caracterizada por poeira nodular difusa nos pulmões como resultado da exposição prolongada a poeiras betuminosas ou antracite nas minas de carvão. Pneumoconiose de mineiros de carvão também chamado preto ou preto doença pulmonar pulmão. Um mineiro de carvão que sofre ou desenvolve uma antracose pode apresentar numerosos nódulos pulmonares arredondados em um curto espaço de tempo. Estes nódulos aparecem às vezes na ausência de uma antracose simples. Histologicamente podem assemelhar-se a nódulos reumatóides, mas têm uma área periférica de inflamação aguda. Esses nódulos representam a resposta imune associada à diátese reumatóide.

A mineração na Colômbia é um fator estratégico economicamente para o desenvolvimento. No entanto, existem fatores negativos decorrent surgindo girando-o em uma corrida sem controle ou regras claras. Esta atividade está pendente no território, arrastando um rastro de problemas na sociedade, o meio ambiente, bem-estar e da saúde das pessoas.

Palavras-chave: Pneumoconiosis, Pulmão negro, nódulos de Carvão, Dispneia, Macrófagos alveolares. 


\section{Introducción}

Neumoconiosis o antracosis se le denomina al grupo de enfermedades pulmonares caracterizadas por inhalación de partículas inorgánicas; término introducido por Zenker en 1867 del griego pneuma: "aire" y kovni (o konis) "polvo" (1). En 1494 ya se hablaba de los efectos perjudiciales del polvo inhalado por los mineros. La primera vez que se diferenció el polvo de origen inorgánico del orgánico como agente etiológico de neumopatías de etiología laboral fue en las descripciones de Charles Thackrah en 1831. Desde Aristóteles y Galeno, los médicos asocian las enfermedades con su profesión u ocupación; por otra parte, Ramazzini recomienda hacer una anamnesis dirigida, averiguando siempre en qué actividad trabaja el enfermo que se examina. Es así el caso de la minería; una de las actividades más antiguas de la humanidad puesto que en épocas prehistóricas, el hombre ya la utilizaba para la fabricación de sus herramientas. Sin la minería sería muy difícil el desarrollo de la humanidad tal y como la conocemos hoy. La extracción de minerales siempre ha constituido uno de los indicadores básicos de las posibilidades de desarrollo económico de un país, y a los minerales descubiertos por el hombre, se le da un valor económico sobresaliente debido a la utilidad que prestan a la humanidad (2).

Pero, también la minería da lugar a un sinnúmero de enfermedades debido a la acumulación de polvo en los pulmones asociada a una reacción patológica (fibrosa) ante su presencia (3). Dichos riesgos han sido reportados desde 1864, de acuerdo con el doctor William Farr, quien demostró mayores tasas de mortalidad en los mineros de carbón de Durham y Northumberland (Inglaterra), en comparación con la población general (4).

De acuerdo a la OMS (World Health Report), en el año 2012 los factores de riesgo ocupacionales contribuyeron con un $1,5 \%$ de los años de vida ajustados en función de la discapacidad (AVAD); además, desde el punto de vista de la mortalidad, a las partículas en suspensión se les atribuye 1,6 de cada 100 muertes, siendo éste el factor de riesgo ocupacional que más aporta al total de muertes (2).

La Fundación Neumológica Colombiana manifiesta que las enfermedades respiratorias ocupacionales son muy frecuentes, ya que el pulmón es el órgano que mayor interacción tiene con agentes ambientales, siendo las más comunes la neumoconiosis por inhalación de polvo orgánico (5).
El carbón es un mineral inerte y no causan daño en el tejido donde se encuentra, a menos que se deposite en grandes concentraciones causando destrucción del tejido pulmonar; algunos autores lo han relacionado con factores predisponentes del cáncer pulmonar, cuando la antracosis es muy acentuada y se produce además destrucción del tejido pulmonar hasta llegar a los diferentes grados de fibrosis de éste órgano (6).

El depósito de polvo en los pulmones es el resultado de un complicado proceso de inhalación, depuración y retención. Únicamente las partículas menores de 5 micras alcanzan el saco alveolar y una parte de ellas son aclaradas mediante el movimiento de la capa fluida que cubre la pared alveolar (surfactante) hacia el bronquiolo terminal, siendo vehiculadas por el transporte mucociliar. Otro mecanismo de depuración es la fagocitosis, realizada por los macrófagos alveolares. Las partículas que logran alcanzar el intersticio pueden ser fagocitadas por los macrófagos y son eliminadas. Las no depuradas y depositadas en el pulmón son las que provocan la reacción patológica como es el caso de la neumoconiosis (7).

\section{La minería en Colombia}

"La minería artesanal y en pequeña escala es muy importante para muchas comunidades pobres, ya que proporciona trabajo de tiempo completo o temporal", con frecuencia representa la oportunidad más promisoria de ingresos y/o la única disponible, aunque genere enfermedad, daño social y ambiental $(8,9)$.

El único mineral en el que Colombia tiene una presencia internacional importante es el carbón a gran escala y solo con el 1,1\% de la producción mundial. La participación de la minería en el producto interno bruto (PIB) de Colombia es reducida (10). En el 2012 el carbón mineral representó un poco más del $1 \%$ del PIB; la suma de los minerales metálicos y los minerales no metálicos, cerca del $0,5 \%$; y el petróleo, cerca del $5 \%$. Por otra parte, las regalías presupuestadas por el Ministerio de Hacienda para los años comprendidos entre el 2012 y el 2022 ascenderían a $\$ 98,3$ billones $(9,11,12)$.

Colombia es el país con mayores reservas de carbón en América Latina y cuenta con recursos potenciales de 16.992 millones de toneladas siendo una fuente generadora de divisas y empleo muy importante. Este mineral se ha ido consolidando como el segundo producto de exportación nacional después del petróleo, considerándose que podría superar sus exportaciones. Entre el año 2009 
y 2013, la producción de carbón en Colombia creció en promedio cerca del 3,28\% anual, con una cifra de 85,5 millones de toneladas para el año 2013. De acuerdo al plan de Desarrollo 2010-2014 es reconocido el sector minero como uno de los cinco sectores que avanzan más rápido que el resto de la economía, denominándose como la locomotora minero-energética $(9,10)$.

Las principales reservas de carbón en Colombia se encuentran en la Costa Atlántica, con aproximadamente el $90 \%$ del carbón térmico, que a su vez corresponde al 98\% del carbón nacional; en el interior del país las reservas corresponden a siete departamentos: Antioquia, Valle del Cauca, Cauca, Boyacá, Cundinamarca, Santander y Norte de Santander (12).

Sin embargo, las condiciones de vida y de salud de la población en las zonas mineras y zonas de influencia de explotación de carbón muestran contextos críticos de pobreza, medidos a través del acceso a bienes y servicios; el acceso al servicio público en la zona rural es muy limitado, además se muestra una situación de seguridad alimentaria precaria, sumado a deficiencias en la red de servicios de salud con limitada capacidad de apoyo preventivo tanto para los trabajadores como para sus familias (13).

El sector de la minería es uno de los más desamparados del país en materia de salud ocupacional; desde las condiciones de explotación, transporte y manejo, las cuales se ven ausentes de condiciones laborales y ambientales que garanticen la protección integral en la salud de los trabajadores (14).

A pesar de las consecuencias que genera la exposición prolongada a partículas de carbón, en el país son pocos los estudios sobre el impacto en la salud; una investigación realizada en trabajadores del El Cerrejón, la mayor mina a cielo abierto a nivel mundial, al norte del departamento de la Guajira, demostró valores significativamente mayores de biomarcadores de genotoxicidad, con respecto a un grupo control no expuesto $(9,15)$. Por otro lado, González y cols., en el 2009, reportaron mediante técnicas de espirometría y oximetría una alta prevalencia de alteración funcional respiratoria en trabajadores de la minería del carbón en Paipa-Boyacá, expresada como patrones de tipo obstructivo, restrictivo y mixto, además, una prevalencia mayor de hipoxemia, con respecto a un grupo control (16).

El Ministerio del Interior a través de la Dirección de Consulta Previa es el organismo encargado de gestionar y dirigir el proceso de consulta previa a las comunidades precedente a la explotación del título minero. Aunque no todos los proyectos mineros deben atravesar por este proceso, se estima que aproximadamente tres de cada diez proyectos están en áreas de interés de comunidades indígenas o negras, por lo que deben ser sometidos al proceso. En Boyacá, Cundinamarca y demás departamentos de actividad minera, los procesos de consulta previa no son aplicables a las comunidades campesinas. En la actualidad no existe una norma que regule dicha consulta, y "al no haber sido reglamentado, debe desarrollarse conforme a la interpretación de la normativa general que impera en cada comunidad étnica" $(9,12)$

En el departamento de Cundinamarca, al norte de la Sabana de Bogotá, se encuentran algunos municipios que son mineros de tradición, en los que la minería de carbón ha sido el centro del desarrollo. El inicio de la regulación en materia de explotación de carbón fue el Decreto 805 de 1947. que fue una regulación completa de las minas contratables, seguido por el Decreto 2514 de 1952, que es el origen de la minería formal vigente a la fecha en Cundinamarca. Estos mineros iniciaron su relación con el Estado a través de la figura de los permisos y con diferentes modificaciones a las normativas convirtiéndose en licencias de explotación y/o contratos según el tema normativo de la época. En la actualidad, en la Sabana de Bogotá se cuenta con 582 títulos mineros y 339 solicitudes mineras en trámite, de los cuales 162 corresponden a carbón (30\%); más del $90 \%$ de las explotaciones trabajan utilizando métodos y maquinaria rudimentaria y alcanzan producciones del orden de 1 a 2,5 ton/hombre por turno, además existen alrededor de 242 millones de toneladas de reservas medidas de acuerdo con el Ministerio de Minas y Energía $(17,18)$.

\section{Citotoxicidad}

Existen diferentes factores de riesgo en el ambiente de trabajo para el desarrollo de neumoconiosis, entre los que se incluyen las condiciones del entorno ya sean espacios abiertos o cerrados; sistemas de ventilación industrial, señalización y sistemas de alarma; las concentraciones de partículas de polvos de carbón y el tamaño de éstas (19).

Siguiendo este concepto, se ha clasificado al carbón con base a su mayor o menor contenido de éste mineral, en primer lugar, se encuentra la antracita, seguido del carbón bituminoso (hulla), el carbón sub-bituminoso y finalmente el lignito (el de más bajo rango). Las minas con un carbón de alto rango presentan mayor contenido de cuarzo y consecuentemente más silice, esto hace que se aumenten los radicales libres y por tanto generan mayor 
efecto citotóxico y respuesta inflamatoria en los trabajadores que lo inhalan $(20,21)$. De igual forma, el carbón de rango superior recién arrancado, contiene partículas de tamaño más pequeñas que el de rango bajo, por tanto presentan mayor facilidad para llegar a las vías respiratorias inferiores (22).

Esta información fue ratificada por un estudio realizado por Bennett y cols., quienes observaron que los mineros que trabajaban en carbones de rango alto tenían mayor prevalencia de neumoconiosis $(21 \%)$, pero una menor exposición al polvo $\left(3,1 \mathrm{mg} / \mathrm{m}^{3}\right)$, mientras que los mineros que trabajaban con carbones de rango bajo, tenían una menor prevalencia de neumoconiosis $(3,9 \%)$ a pesar de su mayor exposición al polvo de carbón $\left(5,5 \mathrm{mg} / \mathrm{m}^{3}\right)(23)$.

Por otro lado, el carbón bituminoso con sus altos contenidos de $\mathrm{FeS}_{2}$ (pirita) tiene gran facilidad para formar de manera espontánea especies reactivas de oxígeno (peróxido de hidrógeno y radicales hidroxilo), produciendo inflamación y degradación del ARN, correlacionándose con una mayor prevalencia a neumoconiosis $(24,25)$. Bajo este concepto de riesgo, se considera entonces que ninguna persona debe estar expuesta a polvo en concentraciones superiores a $10 \mathrm{mg} / \mathrm{m}^{3}$ de polvo total o a $3 \mathrm{mg} / \mathrm{m}^{3}$ de fracción respirable, para 8 horas de trabajo. Además, según las condiciones adoptadas por la American Conference Governmental Industrial Hygienist, la concentración máxima de aire aceptada para 8 horas de trabajo con máximo 40 horas semanales de exposición a monóxido de carbono, es de 25 partes por millón (26).

Sin embargo, el carbón ha dejado huella no solo en los trabajadores de las minas sino también de manera indirecta en las comunidades que viven cerca o que están en los corredores del transporte de este mineral, o de los puertos donde el carbón se carga, ya que inevitablemente el polvillo que se desprende a su paso causa efectos respiratorios por exposición crónica al aire tóxico. De acuerdo a un estudio epidemiológico de la Universidad Nacional sobre la prevalencia de síntomas y enfermedad respiratoria en niños menores de 12 años en una zona carbonífera del departamento de Cesar, se encontró que los niños de los corregimientos cercanos a los depósitos mineros presentaban mayor prevalencia de asma y alguna enfermedad respiratoria aguda. De igual forma, la Universidad de los Andes y el Ministerio del Medio Ambiente encontraron que entre más lejos esté ubicado el hogar de una zona de extracción, menor será el número de casos de enfermedades agudas respiratorias (27-29).

\section{Fisiopatología}

Las neumoconiosis son un grupo de enfermedades que se caracterizan por la afección permanente del intersticio pulmonar, producto del acumulo de polvo inhalado en los pulmones y la reacción tisular que éste provoca. Dependiendo de la sustancia causante hay diferentes tipos de neumoconiosis, como la silicosis (silice cristalina $\mathrm{SiO}_{2}$ ); beriliosis (berilio); siderosis (hierro); silicatosis (caolín, talco, asbesto) y neumoconiosis de la mina de carbón (carbón y $\left.\mathrm{SiO}_{2}\right)(30)$.

El carbón no es un mineral con una composición uniforme; las formas con mayor capacidad de combustión poseen mayor riesgo de producir neumoconiosis del minero de carbón debido a que contienen más radicales libres de superficie. Además, según sus propiedades fisicoquímicas, puede absorber compuestos aromáticos orgánicos ambientales como benceno, metileno y fenol, lo cual puede afectar su actividad biológica (31).

El carbón permanece en forma de aerosol como partícula en el aire durante un tiempo considerable, aunque el sistema respiratorio cuenta con mecanismos para remover estas partículas, especialmente las que se localizan en las vías respiratorias superiores, las partículas de pequeño tamaño pueden depositarse por sedimentación sobre todo en las vías aéreas pequeñas, como bronquiolos terminales y respiratorios; esto puede evidenciarse en un corte pulmonar microscópico en el cual resalta la acumulación de polvo a su alrededor, siendo el fundamento anatomopatológico para el moteado micronodular visible en la radiografía de tórax en la neumoconiosis simple (Figura 1) (32).

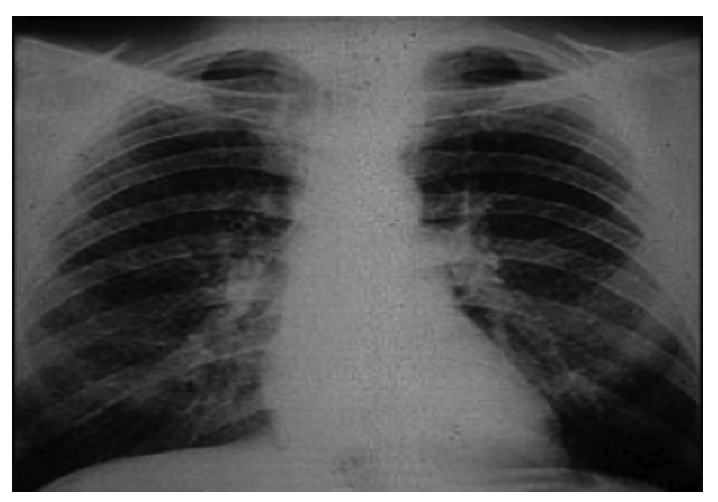

Figura 1. Radiografía de tórax muestra los pulmones de un minero. A ambos lados de los pulmones hay áreas difusas, pequeñas (de 1 a $3 \mathrm{~mm}$. cada una) y claras. Tomado de: https://www.nlm.nih.gov/ medlineplus/spanish/ency/esp_imagepages/1599.htm 
Por otra parte, el patrón de ventilación afecta también la cantidad de aerosol depositado; partículas de tamaño entre $100 \mu \mathrm{m}$ (polvo grueso) y 0,1 $\mu \mathrm{m}$ (humo condensado recientemente) tienen velocidades entre 25 y $0,000085 \mathrm{~cm}$ por segundo; durante el esfuerzo, la velocidad del flujo respiratorio aumenta, con lo que el depósito por impactación se incrementa, y es lo que sucede con los mineros del carbón debido a su duro trabajo físico justo frente a la mina donde las concentraciones de polvo son elevadas, incrementándose los riesgos de inhalación y depósito $(32,33)$.

Según la Comisión Internacional de Protección Radiológica se demuestra que el mayor tamaño de la partícula que logra entrar al tracto respiratorio es aproximadamente de $100 \mu \mathrm{m}$, aquellas de un tamaño menor de $10 \mu \mathrm{m}$ penetran la nasofaringe y las menores de $7 \mu \mathrm{m}$ logran alcanzar los sacos alveolares depositándose en sus recubrimientos $(34,35)$.

En los mineros del carbón pueden presentarse dos tipos de fisiopatologías: una de ellas es la neumoconiosis simple que se caracteriza por la presencia de agregados de partículas pequeñas de carbón compuestas por sustancias dañinas de alta solubilidad alrededor de bronquiolos terminales y respiratorios con una ligera dilatación de estas vías aéreas pequeñas, que pueden producir un daño pulmonar de inicio rápido; esta forma causa escasa incapacidad y deben pasar más de veinte a treinta años para que se haga aparente la lesión pulmonar, ya que éstas progresan muy lentamente; sin embargo la disnea y la tos que se acompañan, pueden estar relacionadas con el antecedente de tabaquismo del minero; esta forma se diagnostica por la presencia de nódulos pequeños de 2 a $5 \mathrm{~mm}$ de diámetro, en los campos pulmonares en las radiografías de tórax (36-38).

Por otro lado, hay una forma más avanzada de la enfermedad, conocida como fibrosis masiva progresiva, en la cual los pulmones se caracterizan por presentar masas condensadas de tejido fibroso negro infiltrado con polvo. A esta forma clínica se llega cuando el minero permanece expuesto a grandes concentraciones de polvo con partículas de tamaño mayor, resistentes a la disolución, las cuales persisten dentro del parénquima pulmonar durante años, produciendo disnea cada vez más intensa lo que puede desencadenar una insuficiencia respiratoria. La mayor parte de la sintomatología se debe a los efectos de bronquitis crónica y enfermedad pulmonar obstructiva crónica inducida por el polvo de carbón. Se caracteriza por nódulos de más de $10 \mathrm{~mm}$ de diámetro $(37,39)$.

El desarrollo de esta patología depende distintas variables como: la cantidad de polvo retenida en los pulmones y vías respiratorias; la forma, tamaño y flotabilidad de las partículas; la solubilidad y reactividad fisicoquímica de la partícula y los posibles efectos añadidos de otros irritantes (como el tabaquismo concomitante) que deterioran el aclaramiento mucociliar ocasionando la acumulación significativa de polvo en los pulmones (40) (Figura 2).

Para la eliminación de las partículas los pulmones cuentan con dos mecanismos: el sistema mucociliar y los macrófagos alveolares. En el primero, las glándulas seromucosas bronquiales contienen células productoras de moco y células serosas, quienes transportan por los conductos el moco a la superficie de las vías aéreas; ésta película de moco está formada por dos capas, una superficial constituida por un gel pegajoso y viscoso que atrapa eficazmente las partículas depositadas, y una capa más profunda menos viscosa, que permite el movimiento de los cilios. Por otro lado, el moco contiene inmunoglobulina A ( $\operatorname{Ig} \mathrm{A})$, proveniente de las células plasmáticas y del tejido linfoide, lo que le permite al cuerpo protegerse frente a proteínas extrañas, bacterias y virus. La depuración de la mucosa bronquial sana puede completarse a las $24 \mathrm{~h}$, sin embargo, en ambientes con mucho polvo, hay una secreción intensa de moco donde la tos y la expectoración ayudan a la depuración (32).

En los alveolos el sistema mucociliar se interrumpe y las partículas pueden ser captadas por las células epiteliales, atravesarlas e interaccionar directamente con los fibroblastos y macrófagos intersticiales; algunas pueden llegar a los vasos linfáticos mediante el drenaje o dentro de los macrófagos que migran iniciando una respuesta inmunitaria consistente en la liberación de factores proinflamatorios y fibrogénicos; además también participan leucocitos, fibroblastos y, posiblemente, mastocitos, eosinófilos y miofibroblastos, que producen sustancias con gran poder destructivo, como son los radicales oxidantes (ROS), las proteasas y otras enzimas. Paralelamente, el sistema antioxidante y antiproteasa se activan, liberándose un conjunto de factores de crecimiento y organización que intentan reparar los tejidos dañados. Cuando los sistemas de defensa son superados, se producen las lesiones propias de la enfermedad, las cicatrices y en definitiva, la fibrosis (41-43). 


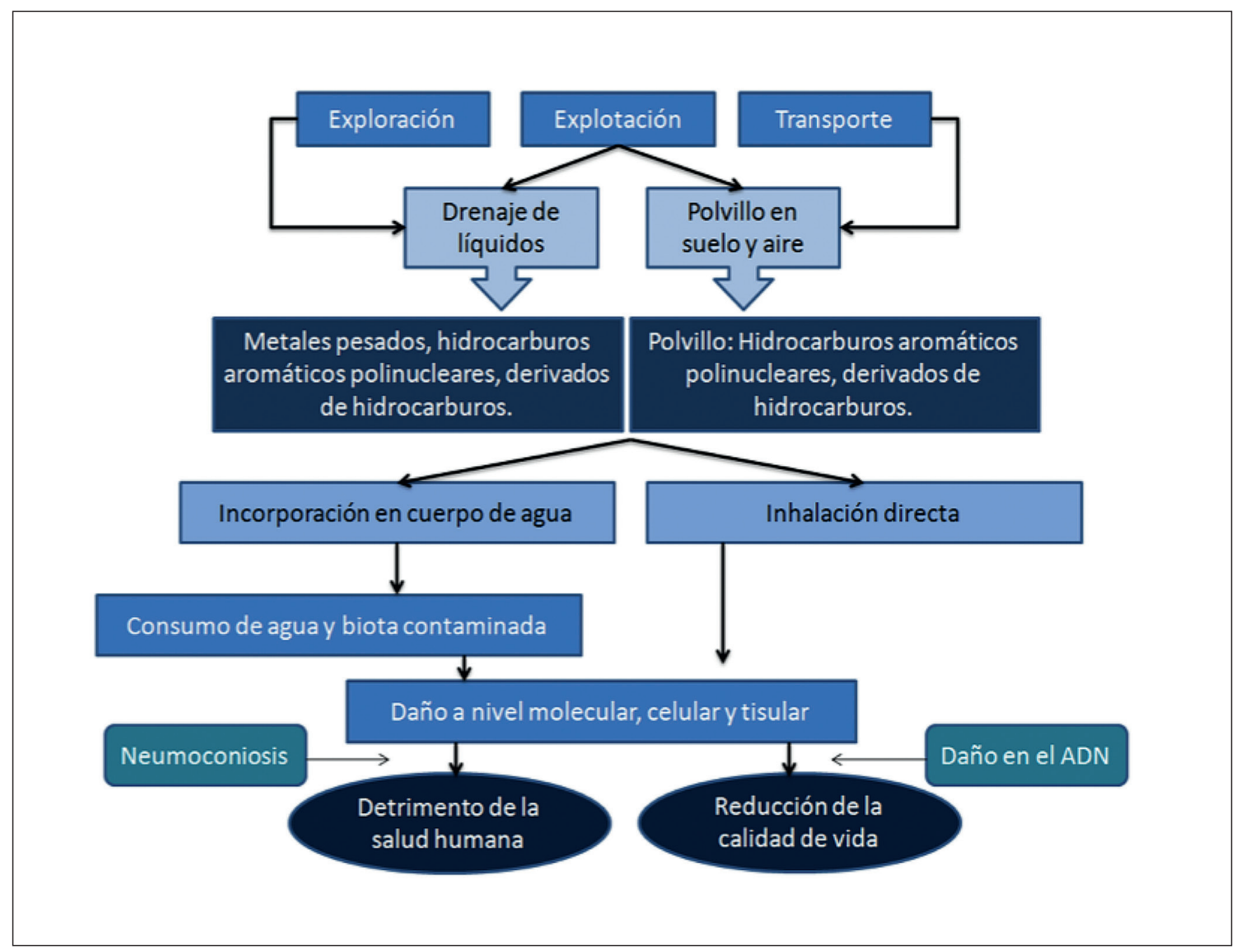

Figura 2. Minería de carbón: efectos sobre la salud humana. Modificado de: Verbel J. Efectos de la Minería en Colombia sobre la salud humana.2011.

La lesión patológica distintiva de la neumoconiosis es la mancha de carbón de $1-2 \mathrm{~mm}$ de diámetro (correspondiente a los macrófagos cargados de este mineral) y los nódulos de carbón que contienen una red delicada de fibras de colágeno producidos en los lóbulos superiores y zonas superiores de los lóbulos inferiores, de característico color negro y aspecto nodular (38).

Por otro lado, muchos mineros presentan enfermedad reumatoide, la cual se asocia con fibrosis nodular de los pulmones; una patología descrita por Caplan y cols., donde se manifiestan los "nódulos de Caplan" cuyo síndrome lleva su mismo nombre, son formaciones similares al nódulo silicótico clásico y al reumatoide, sin embargo, presenta una serie de particularidades morfológicas que son propias relacionadas con ciertos factores inmunológicos que intervienen en su formación $(44,45)$. Tiene su base en la artritis reumatoide (AR), cuyas manifestaciones extra-articulares más frecuentes son la afectación pleuropulmonar y la vasculitis cutánea; se estima un $18 \%$ de mortalidad de la AR es por causa pulmonar. Este síndrome se caracteriza por la aparición de imágenes nodulares redondeadas en ambos campos pulmonares de localización predominantemente periférica y con posibilidad de cavitarse, junto con alteraciones típicas de la neumoconiosis. Se cree que el material inorgánico inhalado podría ser el desencadenante de fenómenos inmunológicos que favorecen el desarrollo de nódulos, bajo el contexto de la $\operatorname{AR}(46,47)$. 


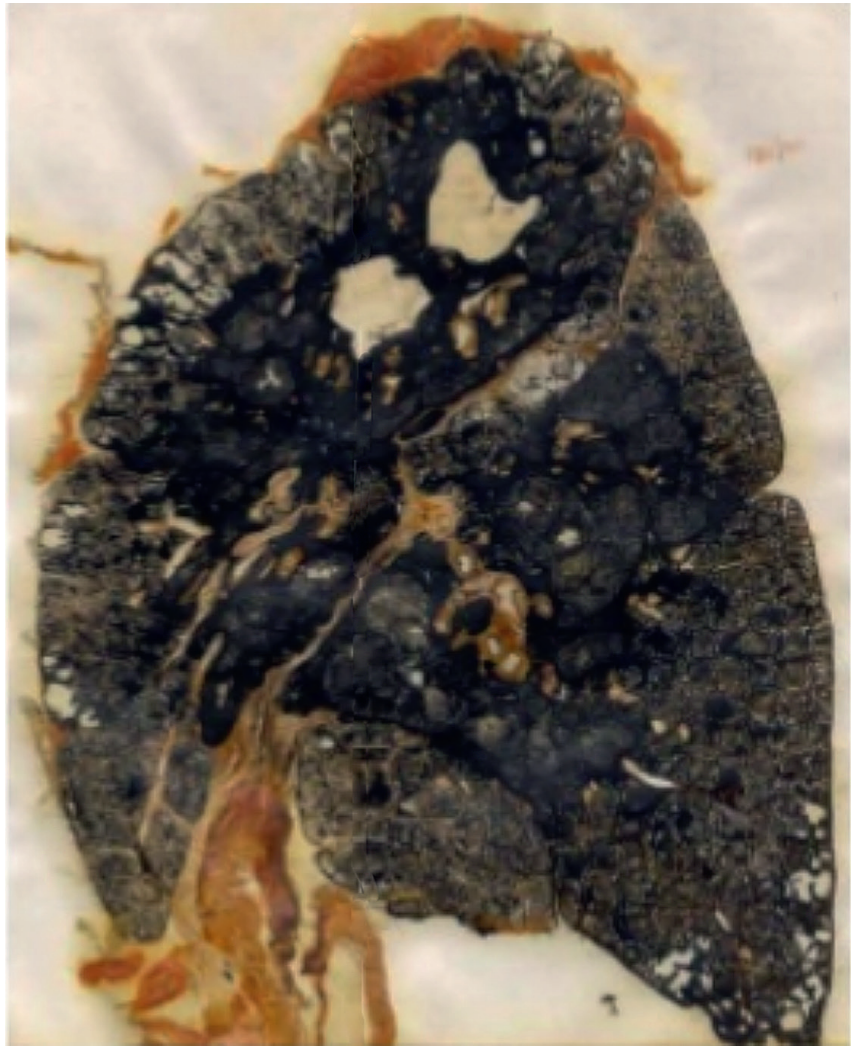

Figura 3. Muestra de patología donde se observa la fibrosis masiva en un minero de carbón. Tomado de: Vázquez M. Neumoconiosis. Enfermedades Laborales. Universidad Juárez del Estado de Durango. Facultad de Medicina.

\section{Apariencia Macroscópica}

A nivel morfológico, la antracosis es la lesión más inofensiva, una vez inhalado, el pigmento del carbón es deglutido por los macrófagos alveolares o intersticiales $y$ es acumulado en el tejido conjuntivo siguiendo a los linfáticos, entre ellos los pleurales (40). Esta se observa en forma de puntilleo fino de color negruzco a gris entre el parénquima, los depósitos del pigmento se observan comúnmente en la parte dorsal de los lóbulos, así como en entre los septos del mismo dando un aspecto grecado; en los linfonodos se puede observar este puntilleo en la corteza siendo los linfonodos más comunes los del árbol respiratorio (48) (Figuras 3 y 4).

En la neumoconiosis complicada, que por lo general se produce sobre una enfermedad de base simple, se caracteriza por múltiples cicatrices intensamente ennegrecidas de $1 \mathrm{~cm}$ o mayores, incluso hasta $10 \mathrm{~cm}$ de diámetro máximo (40).

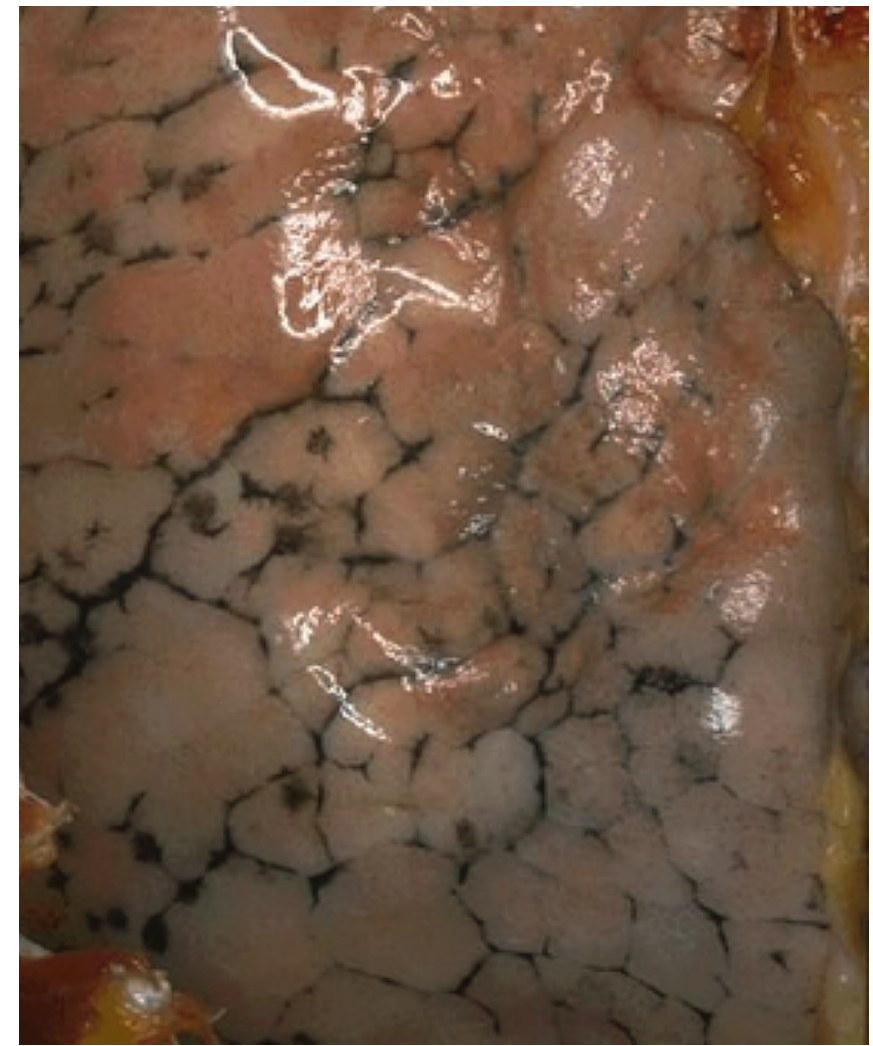

Figura 4. Antracosis-Pulmón. Las líneas oscuras que se ven entre los lóbulos de pulmón bajo la superficie pleural debido a pigmentos antracóticos.

Tomado de: $h t t p: / / w w w . c a n i n u m . c o m / a t l a s / l e s i o n-c e l u l a r / a n t r a c o-$ sis-pulmon.htm

\section{Apariencia Microscópica}

El carbón aparece en forma de diminutos gránulos de color negruzco entre las células del parénquima pulmonar, en la pared alveolar o bien, se encuentra definido en el citoplasma principalmente de macrófagos. En los linfonodos se puede apreciar entre las células linfoides, además, por medio de las ramas linfáticas pueden migrar hacia otros linfonodos cercanos. Sin embargo se localizan principalmente en zonas adyacentes a los bronquiolos respiratorios, el lugar donde el polvo se acumula inicialmente; con el tiempo, se produce dilatación de los alveolos adyacente, dando lugar en ocasiones a un enfisema centrolobulillar. Cuando la enfermedad ha progresado a una neumoconiosis complicada, las lesiones consisten en colágeno denso y pigmento; el centro de la lesión es por lo general necrótico como consecuencia de la isquemia local $(40,49)$ (Figuras 5 y 6). 


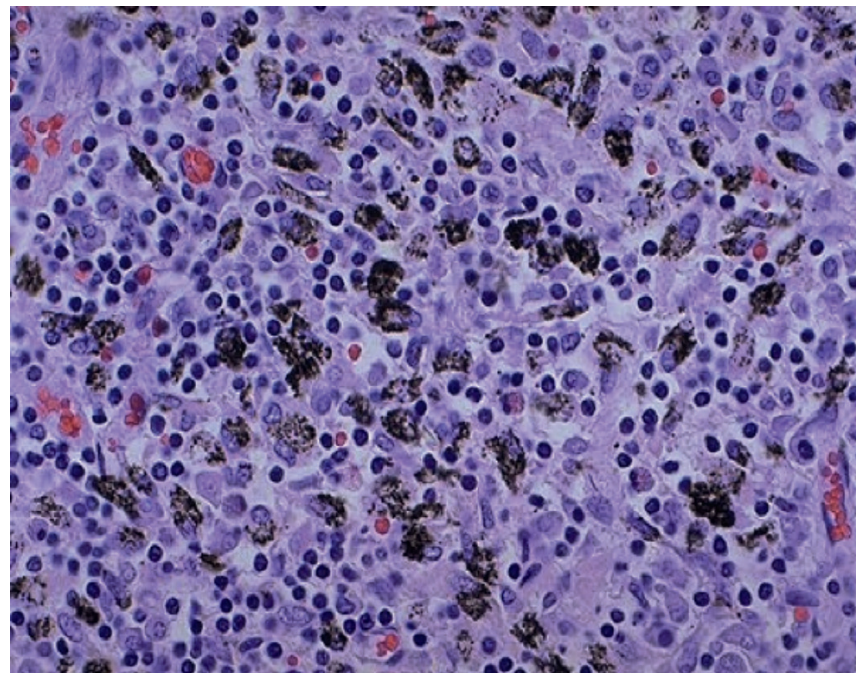

Figura 5. Pigmento antracótico se encuentra en macrófagos ubicados en un linfonódulo. Se observan diminutos puntos pardo-negruzcos en zona de tejido conectivo alrededor de los vasos, intracelulares (en macrófagos) o en las paredes de los alvéolos. Tomado de: http://www.caninum.com/atlas/lesion-celular/antracosis-pulmon.htm

\section{Diagnóstico}

La evolución de la neumoconiosis es lenta y progresiva, en sus etapas iniciales surge silenciosamente y en la mayoría de los casos el diagnóstico se lleva a cabo cuando las complicaciones y secuelas pulmonares están presentes. El diagnóstico y seguimiento de la neumoconiosis se basa en un cuadro clínico y radiológico compatibles con la historia clínica y laboral del paciente, en el cual se evidencian lesiones simples (lesiones radiológicas menores de $10 \mathrm{~mm}$ de diámetro) o complicadas (lesiones mayores de $10 \mathrm{~mm}$ de diámetro). Además se observan pequeñas opacidades redondeadas en el parénquima pulmonar de predominio en los lóbulos superiores. En aquellos casos donde no es posible establecer el diagnóstico por vía clínica o radiológica, es necesario realizar examen histológico de pulmón, un procedimiento invasivo como la broncoscopia, que permite la comprobación de pigmentos y macrófagos presentes $(50,51)$

En los hallazgos clínicos de la neumoconiosis simple, puede encontrarse pocos síntomas respiratorios con un examen radiológico anormal y las pruebas funcionales respiratorias casi siempre normales; con el paso de los años y al avanzar la enfermedad pueden aparecer síntomas como tos y dificultad respiratoria, que pueden desencadenar "cor pulmonare" y falla cardiaca derecha (52).

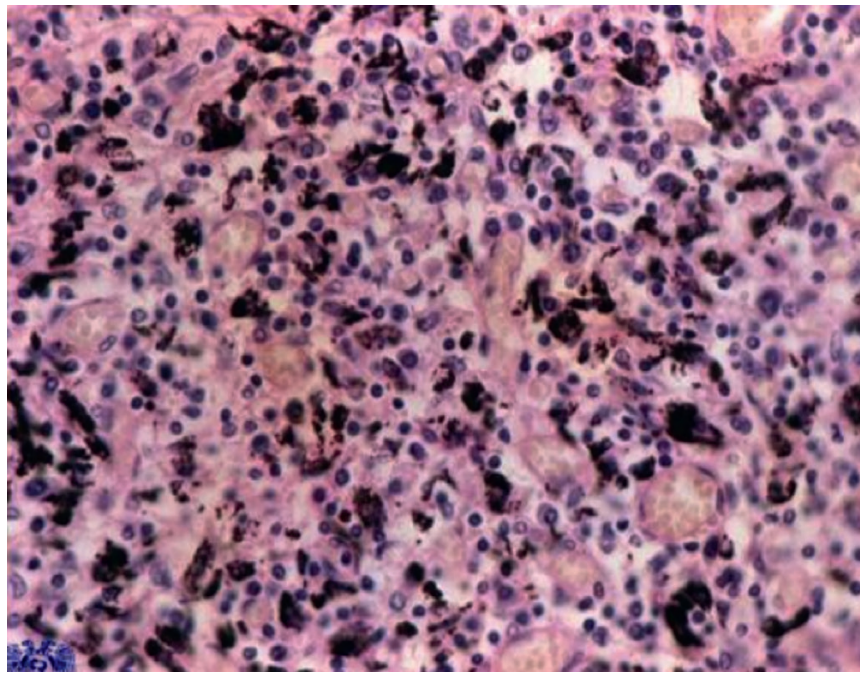

Figura 6. Corte de ganglio linfático traqueal con antracosis. Depósito de carbón en el citoplasma de los macrófagos como inclusión exógena. Créditos Fotografía: Dr. Joaquín Carrillo Farga Laboratorio de Histología, DBCT, FACMED, UNAM

\section{Recomendaciones finales y medidas de prevención en neumoconiosis}

Pese a que la extracción de carbón genera un crecimiento acelerado del producto en las regiones mineras, se suelen presentar graves retrasos en la generación de condiciones de bienestar en la población, restringiendo notablemente el desarrollo humano local; paradójicamente se observan los altos niveles de pobreza, en medio de una actividad que genera enormes riquezas (53).

Es indudable que uno de los factores de riesgo al que ha estado expuesta la humanidad desde tiempos inmemoriales es el material particulado del carbón, uno de los contaminantes atmosféricos más sobresalientes a nivel mundial. Es ante esta situación a la que se ven enfrentados miles de colombianos trabajadores en las minas, ausentes de amparo, garantías laborales y de salubridad; por ello es importante generar una conciencia en torno a la prevención de la enfermedad, partiendo desde los empleadores, quienes refuercen las actividades de vigilancia en salud de sus trabajadores; implementando estrategias eficaces y desarrollando programas de capacitación que generen conocimiento del factor de riesgo, la necesidad del uso correcto de los elementos de protección, la importancia del autocuidado y sobretodo la invitación al abandono del hábito tabáquico $(54,55)$. 
El uso de elementos de protección respiratoria que sean garantizados y certificados por las entidades competentes es fundamental, al igual que los programas de vigilancia médica como una historia clínica estandarizada con énfasis en antecedentes ocupacionales, un examen físico enfocado en el sistema respiratorio, radiografía de tórax y espirometría; con seguimiento y evaluación periódica de las condiciones laborales y médicas del trabajador (19) (Figura 7).
Por otra parte, los marcadores de predicción y el descubrimiento temprano de neumoconiosis son indispensables para la aplicación oportuna de estrategias de intervención, como lo reporta González y cols., con el uso de pruebas de función respiratoria, de bajo costo, fácil ejecución y de gran utilidad para el diagnóstico precoz y control en trabajadores mineros expuestos al polvo de carbón (16).

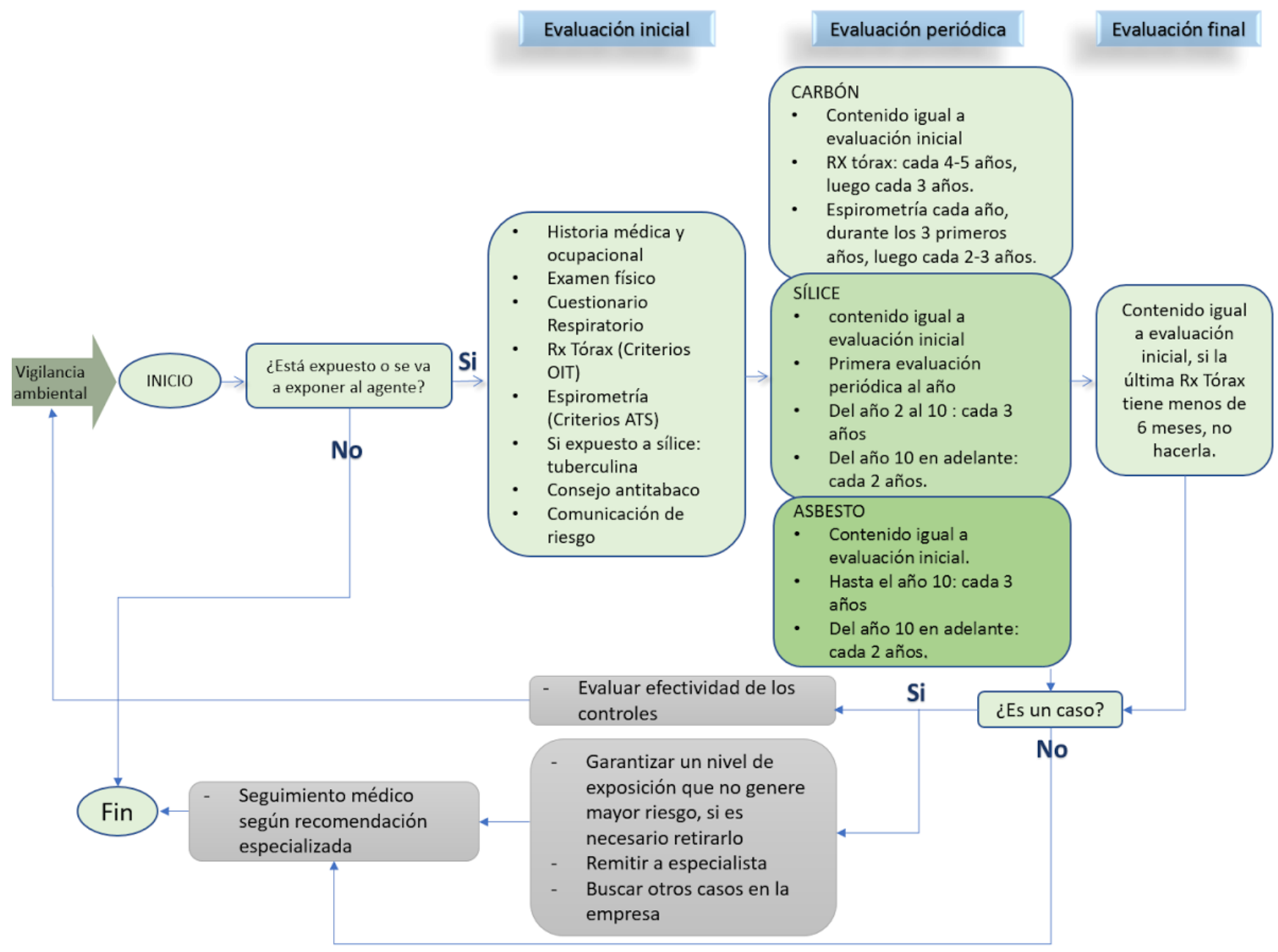

Figura 7. Vigilancia médica ante la exposicón a Carbón, sílice y asbestos. Tomado de: Ministerio de Protección Social Republica de Colombia. Plan Nacional para la prevención de la silicosis, la neumoconiosis de los mineros de carbón y la asbestosis 2010-2030. Julio de 2011. 


\section{Conflicto de intereses}

Ninguno

\section{Referencias}

1. Sociedad Mexicana de Radiología e Imagen, A.C. XLIV Curso Anual de Radiología e Imagen. XXII Encuentro Nacional de Residentes. Neumoconiosis. 2010.

2. ACGIH. American Conference Governmental Industrial Hygienist. TLVs and BEIs. Cincinnati, Ohio, 2002.

3. Fraser R., Pare J., Pare P., Fraser R., Genereux G. Pleuropulmonary disease caused by inhalation of inorganic dust. En: Diagnosis of diseases of the chest. 3rd edn. Filadelfia: W.B. Saunders. 1990; 3:2276-2381

4. Farr W, Buck C, Llopis A, Nájera E, Terris M. Mortalidad de los mineros: Una selección de los informes y escribos de William Farr. En: El desafío de la Epidemiología. Problemas y lecturas seleccionadas. Parte I, Desarrollo Histórico. Organización Panamericana de la Salud. 1988.p.69

5. Zapata R., Pérez J., Salazar C. Carbones colombianos: clasificación y caracterización termoquímica para aplicaciones energéticas. Revista ION. ISSN 2145-8480.

Disponible en: <http://revistas.uis.edu.co/index.php/revistaion/article/view/4589/5157>. Fecha de acceso: 18 jun. 2016.

6. Verbel J. Efectos de la minería en Colombia sobre la salud humana. Unidad de Planeación Minero Energética (UPME). 2011.

Disponible en: http://www1.upme.gov.co/sites/default/ files/forum_topic/3655/files/efectos_mineria_colombia sobre salud humana. pdf

7. Green G., Jakab G., Low R., Davis G. Defense mechanisms of respiratory membrane. Am Rev Respir Dis. 1977; 115:479-514.

8. IIED: International Institute for Environment and Development 2012, p.447

9. León Rodríguez N.R. Estudio sobre competitividad de la minería de carbón del interior en Colombia, aplicando el modelo de Competitividad Sistémica del Instituto Alemán de Desarrollo (GDI). Universidad Nacional de Colombia. Facultad de Ingeniería, Departamento de Ingeniería Industrial. Tesis de Grado 2016.

10. Unidad de Planeación Minero Energética, Ministerio de Minas y Energía, República de Colombia. Indicadores de la minería en Colombia. Bogotá, D.E. 2014.

11. Unidad de Planeación Minero Energética, Ministerio de Minas y Energía, República de Colombia. Plan Nacional de Desarrollo Minero al 2014. Sector minero de cara a la sociedad. 2012.

12. Unidad de Planeación Minero Energética. Ministerio de Minas y Energías. La cadena del Carbón. El carbón colombiano. 2005

13. Ruiz F., Amaya J., Peñaloza R., Ferro M., Cárdenas M., Quiroga A. Calidad de vida y salud: un diagnóstico de la zona de influencia de Cerrejón. Cerrejón Minería responsable, Cendex de la Universidad Javeriana. Primera edición, ECOE Ediciones. 2011. Bogotá.

14. Ospina J., Manrique F., Garzón J. Salud y trabajo: minería artesanal del carbón en Paipa, Colombia. Avances en Enfermería. 2010;28(1):107-15.

15. León G., Espitia L., Hoyos L., Da silva J., Hartmann A., Pegas J., Quintaja M. Assessment of DNA damage in coal open-cast mining workers using the cytokinesis-blocked micronucleus test and the comet assay. Science of The Total Environment. 2011;15(4):686-691.

16. González N., Manrique F., Ospina J., Roa M., Hurtado E. Utilidad De Las Técnicas De Espirometría Y Oximetría En La Predicción De Alteración Pulmonar En Trabajadores De La Minería Del Carbón En Paipa- Boyacá. Rev.Fac. Med.2009;57(2):100-110.

17. Federación de Productores de Carbón de Cundinamarca. Impacto socioeconómico de la minería de carbón en Cundinamarca. 2013. Disponible en: http://fedecundi.com/ resumen.pdf

18. Montoya D., Reyes G. Ministerio de Minas y Energía. INGEOMINAS. Geología de la Sabana de Bogotá. 2005.

19. Ministerio de la Protección Social. Guía de atención integral basada en la evidencia para neumoconiosis (silicosis neumoconiosis del minero de carbón y asbestosis). Imprenta Nacional de Colombia. 2007. ISBN 978-958-98067-3-9

20. Zapata M. Perspectiva Neumológica-Silicosis. 2011;11(2). Disponible en: http://www.neumologica.org/enfermedades\%20ocupacionales.htm

21. Gamble J., Regen R., Glenn R. A critical review of coal worker's pneumoconiosis (CWP) and coal rank for evaluation of safe exposure levels in coal mining. J Clinic Toxicol. 2012; S1:009. Doi:10.4172/2161-0495.S1-009.

22. Dalal N., Suryan M., Vallyathan V., Green F., Jafari $\mathrm{B}$, Wheeler R. Detection of reactive free radicals in fresh coal mine dust and their implication for pulmonary injury. Ann Occup Hyg. 1989;33(1):79-84.

23. Bennett J, Dick JA, Kaplan YS, Shand PA, Shennan DH, et al. The relationship between coal rank and the prevalence of pneumoconiosis. Brit J Ind Med. 1979; 36:206210

24. Huang X., Li W., Attfield M., Nádas A., Frenkel K., et al. Mapping and prediction of coal workers' pneumoconiosis with bioavailable iron content in the bituminous coals. Env Health Perspect. 2005; 113:964-968.

25. Cohn C, Richard L, Sanford S, Thomas O, Martin S. Role of pyrite in formation of hydroxyl radicals in coal: possible implications for human health. Part Fibre Toxicol.2006;3:16-26.

26. ACGIH. Guide to Occupational Exposure Values. Compled by ACGIH. Cincinnati. OHIO. 2005.

27. Melo D., Peña R., Rocha J., Torres A. El carbón de Colombia ¿quién gana y quién pierde? Minería, comercio global y cambio climático. Estudios de caso departamentos Cesar y Magdalena. Editora 3 Ltda. 2015. p.146.

28. Quiroz L., Hernández L., Agudelo C, Medina K., Robledo R., Osorio S. Enfermedad y síntomas respiratorios en 
niños de cinco municipios carboníferos del Cesar, Colombia. Rev. Salud Pública. 2013;15(1):66-79.

29. Mendieta J., Perdomo J., Rodríguez M., García L., Rodríguez O., Cardona D., et al. Valoración Económica Ambiental En La Zona Carbonífera Del Cesar Que Comprende Los Municipios De Becerril, Agustín Codazzi, Chiriguaná, El Paso Y La Jagua De Ibirico. Universidad de los Andes. Ministerio de Ambiente, Vivienda y Desarrollo Territorial. 2010

30. Martínez A, Aguilar T. Impacto socioeconómico de la Minería en Colombia. Fedesarrollo Centro de Investigación Económica y Social. 2012.

31. Guthrie G. D. Geology and Geochemistry Group. Mineral Properties and Their Contributions to Particle Toxicity. Los Alamos National Laboratory, Environ health Perspect 1997;105(5):1003-1011

32. West J. Fisiología y fisiopatología pulmonar. Estudio de casos. Segunda edición. 2008.p.125-136.

33. MINERCOL. Ministerio de minas y Energía. Ministerio de Transporte. Ministerio de ambiente, vivienda y desarrollo territorial. Guía ambiental transporte de Carbón. 2004.

34. Nieto O. Material Particulado: Efectos en la Salud. En Contaminación del Aire por Material Particulado. Zonas Urbanas, Complejos Industriales o Mineros. Dispersión y Monitoria. AINSA. Medellín. Octubre 1993.p.33-47.

35. García H. Evaluación del riesgo por emisiones de partículas en fuentes estacionarias de combustión. Estudio de caso: Bogotá. Universidad Nacional de Colombia. 2006.p.3-10.

36. Martínez C. Neumoconiosis. Rev Patol Respir. 2005;8(1):43-44.

37. Stevens A., Lowe J., Scott I. Patología clínica. Tercera edición. Editorial Manual Moderno. 2011.

38. Arango M., Sánchez P., Ríos A. Neumoconiosis del minero de carbón. Universidad Ces. Facultad de medicina. 2012.

39. Beltran D., Manotas R., Varona M., Idrovo A., Torres C. Síntomas Respiratorios Y Neumoconiosis Por Polvo De Carbón Diagnosticada Radiológicamente, En Los Trabajadores De Minería Subterránea En Boyacá En El Año 2014. Disponible en: http://repository.urosario.edu.co/ bitstream/handle/10336/9647/327865252014.pdf?sequence $=1$

40. Kumar V., Abbas A., Aster J. Robbins y Cotran. Patología Estructural y Funcional. Novena Edición. ELSEVIER. 2015.

41. Davis G. Pathogenesis of silicosis: current concepts and hypotheses. Lung. 1986; 164:139-154.

42. Brooke T, Churg M, Churg A. Mechanisms in the pathogenesis of asbestosis and silicosis. Am Rev Respir Crit Care Med. 1998; 157:1680-1698.
43. Schins R, Borm P. Mechanisms and mediators in coal dust induced toxicity: a review. Ann Occup Hyg. 1999; 43:7-33.

44. Caplan A. Certain unusual radiological appearances in the chest of coal-miners suffering from rheumatoid arthritis. Thorax. 1953; 8:29-37.

45. Díaz J., Camp R. Rehabilitación en la arthritis Reumatoide. Editorial Masson. Barcelona. 2002. p.p.18.

46. Mial W., Caplan A., Cochrane A., Kilpatrick G., Oldham M. An epidemiological study of rheumatoid arthritis associated with characteristic chest X-ray appearances in coal-workers. Br Med J.1953;2(4848):1231-1236.

47. Hitado J, Escribano D, Roman A, Méndez A, Mosquera JA. Lesiones pleuropulmonares en las enfermedades reumáticas. Repercusión sobre la neumoconiosis del carbón. Rev Esp Reumatol, 1979; 6: 187-237.

48. Torres C. Neumología. 6a ed. Medellín Colombia: Corporación para Investigaciones Biológicas; 2007.

49. Yeoh C., Yang S. Pulmonary function impairment in pneumoconiotic patients with progressive massive fibrosis. Chang Gung Medical Journal. 2002;25(2):72-80.

50. Xaubet A., Ancochea J., Blanquer R., Montero C., Morell F., Rodríguez E., et al. Diagnóstico y tratamiento de las enfermedades pulmonares intersticiales difusas. Arch Bronconeumol. 2003;39(12):580-600.

51. Martínez C., Quero A., Isidro I., Rego G. Enfermedades pulmonares profesionales por inhalación de polvos inorgánicos. La medicina hoy. Hospital Central de Asturias. Oviedo. 2001; 61:34-39.

52. Bauer T., Heyer C., Duchna H., Andreas K., Weber A., Schmidt E., et al. Radiological findings, pulmonary function and dyspnea in underground coal miners. Respiration; International Review of Thoracic Diseases. 2007; 74(1):80-7.

53. Rudas G. Notas sobre la minería de carbón a gran escala en Colombia. En: La minería de carbón a gran escala en Colombia: impactos económicos, sociales, laborales, ambientales y territoriales. Friedrich-Ebert-Stiftung (FES). Análisis. 2014.

54. Báez A., Bocanegra J. Medidas de intervención para promoción de conductas saludables y prevención de riesgos laborales relacionados con accidentalidad y enfermedad laboral en trabajadores de minas de Carbón. Tesis de Grado. Facultad de medicina. Universidad del Rosario. 2015.

55. Morrice E., Colagiuri R. Coal mining, social injustice and health: A universal conflict of power and priorities. Health \& Place. 2013; 19:74-79. 